\title{
DESIGNS AND MEASUREMENTS OF PROTOTYPE MAGNETS FOR THE HIGH-BRILLIANCE SYNCHROTRON LIGHT SOURCE AT THE UNIVERSITY OF TOKYO
}

\author{
T. Koseki ${ }^{\dagger}$, K. Shinoe, K. Harada, Y. Kamiya\#, H. Kudo, N. Nakamura, \\ T. Shibuya, H. Takaki and Y. Takiyama, \\ The Institute for Solid State Physics (ISSP), the University of Tokyo, Kashiwa 277-8581, Japan \\ H. Inoue and K. Kuno, Mitsuibishi Electric Corporation, Japan
}

\begin{abstract}
A high-brilliance VUV and soft X-ray (SX)

synchrotron radiation (SR) source is being planned to construct at the University of Tokyo. Designs of dipole, quadrupole and fast steering magnets for the storage ring are presented in this paper. Results of prototype measurements of the dipole and steering are also described.
\end{abstract}

\section{INTRODUCTION}

The University of Tokyo has been promoting a project to construct a high-brilliance VUV-SX SR source. The storage ring, called Super SOR [1], has a racetrack shape with a circumference of about $250 \mathrm{~m}$ and can be operated at the beam energies between $1.0 \mathrm{GeV}$ and $1.6 \mathrm{GeV}$. The ring is composed of two long $(29 \mathrm{~m})$ straight sections, two short $(2 \mathrm{~m})$ straight sections, eight matching sections and 20 normal cells. The emittance is $0.75 \mathrm{~nm} \cdot \mathrm{rad}$ at 1.0 $\mathrm{GeV}$ in the ultra-low emittance mode [2,3]. One of the long straight sections is slightly undulated by dipole magnets to configure a saw-tooth shape, which makes possible simultaneous use of four insertion devices installed there. In another long straight section, 27-m long undulator will be installed to generate extremely brilliant $\mathrm{SR}$ in the VUV-SX region [4].

The lattice of the Super SOR ring requires 148 quadrupoles, 72 sextupoles, 28 dipoles for arc sections (20 for the normal cells, eight for the matching sections) and seven dipoles for the saw-tooth sections. In addition, more than 100 steerings for fast orbit feedback and about 30 of DC steerings for COD correction will be installed.

In this paper, we describe designs and prototype models of quadrupole, fast steering and dipole for the arc section. The shapes of dipole and quadrupole have been optimized using 2D programs LINDA and POISSON to obtain good field uniformity. All the prototypes have been built by Mitsubishi Electric Corporation.

\section{DIPOLE MAGNET}

The main parameters of the dipole are listed in Table 1. B and BH denote the dipoles for normal cells and matching sections, respectively. They have an identical cross section (Fig. 1), but core length of $\mathrm{BH}$ is one-half of

\footnotetext{
† koseki@issp.u-tokyo.ac.jp

\# Present address: High Energy Accelerator Research Organization (KEK), Tsukuba, Ibaraki 305-0801, Japan
}

B. A magnetic field of $1.75 \mathrm{~T}$ is required for $1.6-\mathrm{GeV}$ operation. In order to obtain good field uniformity for such a high field, the corners of the pole edges are largely cut off as shown in this figure.

Table 1: Parameters of the dipoles in the arc sections.

\begin{tabular}{|l|c|c|}
\hline & B & BH \\
\hline Bending angle [degree] & 15 & 7.5 \\
\hline Bending radius [m] & 3.056 & 3.056 \\
\hline Core length [m] & 0.8 & 0.4 \\
\hline Maximum field [T] & 1.75 & 1.75 \\
\hline Turns / pole & 34 & 34 \\
\hline Maximum current [A] & 1100 & 1100 \\
\hline
\end{tabular}

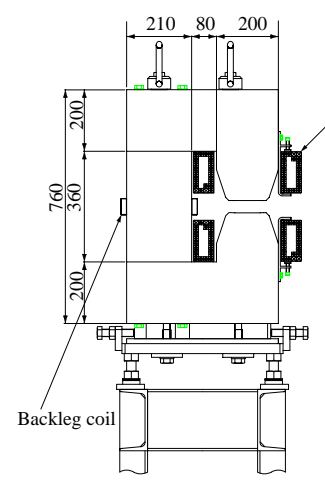

Main coil

Figure 1: Cross section of the dipole.

The prototype of dipole has rectangular configuration and core length of $0.6 \mathrm{~m}$. It is made of forged low-carbon solid-steel. Magnetic field measurement has been carried out using a computer-controlled 3D mapping system with a Hall probe unit (SERIES-9900 gaussmeter, F.W. BELL). An NMR system (PT2025, METROLAB) has been also used to obtain the absolute field strength and calibrate the Hall probe system.

The excitation curve measured at the center of the magnet is shown in Fig. 2. Field strength at 1.0 and 1.6 $\mathrm{GeV}$ operations are indicated in this figure. At the field higher than $1.4 \mathrm{~T}$, the magnet begins to saturate and discrepancy between measured data and 2D calculation becomes large. Because of the short core length, 3D effect is not small even at the position of longitudinal center. The measured data are well reproduced by 3D calculation.

Figure 3 shows the horizontal field uniformity in the center of the magnet at the excitations for $1.0 \mathrm{GeV}(1.10$ 
$\mathrm{T})$ and $1.6 \mathrm{GeV}(1.75 \mathrm{~T})$. The measured data agree well with the $2 \mathrm{D}$ calculation.

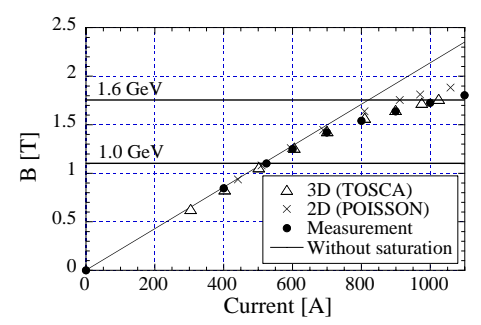

Figure 2: Excitation curve of the dipole prototype.

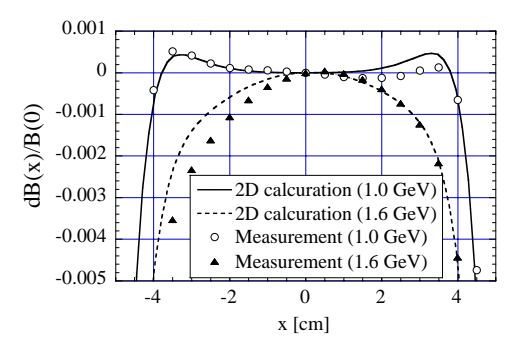

Figure 3: Field uniformity of the dipole prototype.

Field mapping on the vertical median plane has been carried out at intervals of $5 \mathrm{~mm}$ and $10 \mathrm{~mm}$ in the horizontal and longitudinal directions, respectively. In this measurement, both the magnet ends were shimmed with iron plates to reduce the sextupole field component. The integrated field along the nominal beam trajectory was calculated from the mapped data. The effective lengths were measured to be $0.659 \mathrm{~m}$ for the excitation of $1.10 \mathrm{~T}$ and $0.643 \mathrm{~m}$ for $1.75 \mathrm{~T}$. Horizontal uniformity of the integrated field at $1.10 \mathrm{~T}$ and $1.75 \mathrm{~T}$ are shown in Fig. 4. The fields were calculated along the curves parallel to the central trajectory. At $1.10 \mathrm{~T}$, the uniformity inside \pm $30 \mathrm{~mm}$ is within $\pm 5 \times 10^{-4}$, the specified limit required for the ultra-low emittance mode. Although the uniformity is not quite good at $1.75 \mathrm{~T}$, it will be allowable because the operating mode at $1.6 \mathrm{GeV}$ has a large dynamic aperture to the field error.

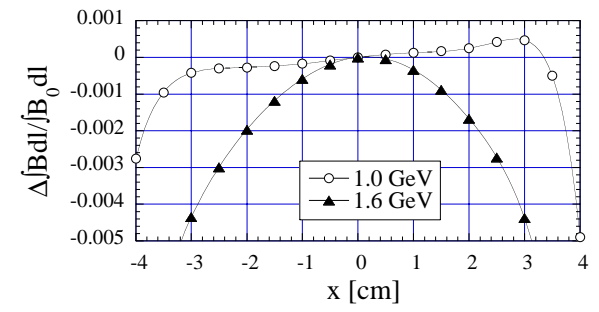

Figure 4: Integrated field uniformity of the dipole prototype.

\section{QUADRUPOLE MAGNET}

For all quadrupoles, C-type profile is adopted to accommodate SR beamline. The magnet core consists of symmetrical upper and lower parts, which are joined by stainless-spacers. The magnet core of all quadrupole has the same cross section, but the stainless-spacers should be designed variously to avoid interference with SR beamlines. The main parameters of the quadrupoles are listed in Table 2.

Figure 5 shows a prototype model of the quadrupole. The core is made of $0.5 \mathrm{~mm}$ laminated silicon-steel and assembled by gluing without supporting plate on each end. The stainless spacer has a hole of $190 \mathrm{~mm} \phi$, to which a pumping port of beam duct can be attached [5]. Figure 6 shows calculated field gradient uniformity of the quadrupole at various excitation levels. Field measurement of the quadrupole prototype is now in progress using newly fabricated harmonic coil system.

Table 2: Parameters of the quadrupole magnet.

\begin{tabular}{|l|c|}
\hline Number of magnets & 148 \\
\hline Bore radius [mm] & 70 \\
\hline Core length [m] & $0.2,0.3,0.35,0.4$ \\
\hline Maximum field gradient [T/m] & 20 \\
\hline Turns / pole & 25 \\
\hline Maximum current [A] & 500 \\
\hline
\end{tabular}
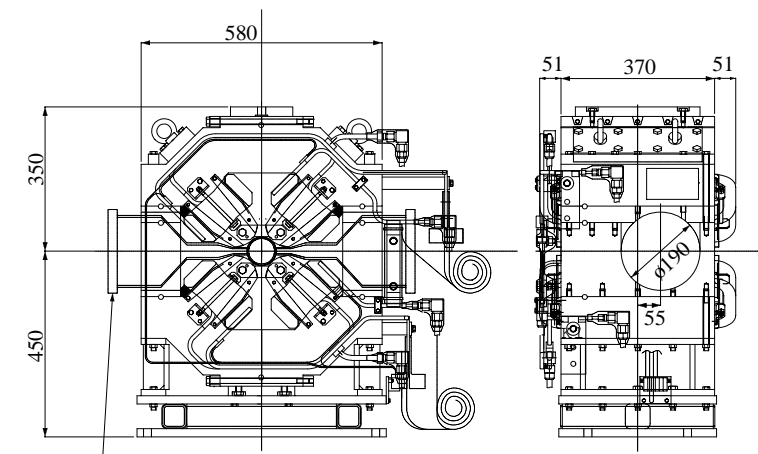

Pumping port

Figure 5: Front and side views of the quadrupole prototype. Two pumping ports are also shown.

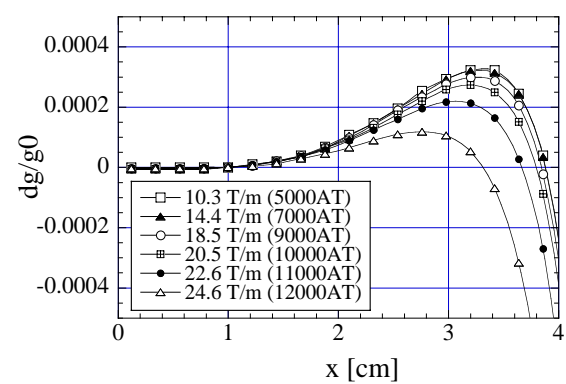

Figure 6: Calculated field gradient uniformity of the quadrupole.

\section{FAST STEERING MAGNET}

In the Super SOR ring, VME-based fast feedback system is applied to beam orbit stabilization [6]. A prototype model of the fast steering for the orbit correction is shown in Fig. 7. It has a square aperture of $119 \mathrm{~mm} \times 119 \mathrm{~mm}$ and can provide both horizontal and vertical fields. The magnet core is composed of four glued stacks of $0.5 \mathrm{~mm}$-thick silicon-steel lamination. The steering will be used not only for the fast feedback but 
also for COD correction. The parameters of the fast steering are listed in Table 3.

Field measurement of the steering prototype has been carried out by the Hall probe system. The effective length of the steering was measured to be $166 \mathrm{~mm}$ at a DC current of $4.2 \mathrm{~A}$. The corresponding deflection angles are $0.11 \mathrm{mrad}$ at $1.0 \mathrm{GeV}$ and $0.07 \mathrm{mrad}$ at $1.6 \mathrm{GeV}$.

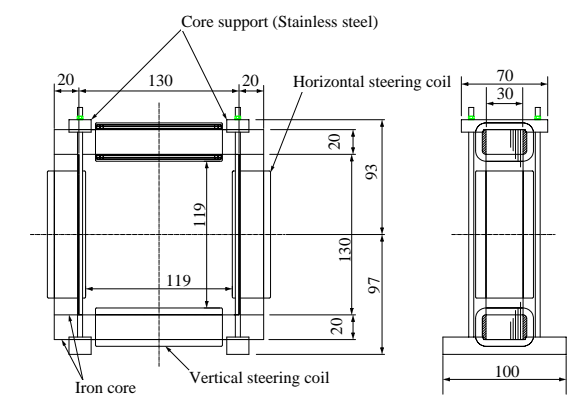

Figure 7: The fast steering magnet.

Table 3: Parameters of the fast steering magnet.

\begin{tabular}{|l|c|}
\hline Maximum field strength [G] & 30 \\
\hline Core length [mm] & 30 \\
\hline Turns / pole & 76 \\
\hline Maximum current $[\mathrm{A}]$ & 5 \\
\hline Coil resistance $[\mathrm{m} \Omega]$ & 137 \\
\hline Coil inductance $[\mathrm{mH}]$ & 3 \\
\hline
\end{tabular}

A good frequency response up to $2 \mathrm{kHz}$ is required to the steering. For the Super SOR ring, most of the beam ducts will be made of aluminum except for the sections of insertion devices, pulse magnets, rf cavities, bellows ducts and so on [5]. If the fast steering is installed at the aluminum beam duct, the frequency response is deteriorated by the effect of eddy current induced on the duct [7]. Therefore, in the Super SOR ring, all of the steerings will be installed at the bellows ducts, which made of stainless steel, so as not to be affected by the eddy current.

We have fabricated the prototype model of the bellows duct, which has copper-beryllium spring fingers [5]. The frequency response of the steering has been measured with the bellows duct. As an AC power supply, we adopt a bipolar switching-mode converter with switching frequency of $100 \mathrm{kHz}$ (IPM-BP series, IDX Corporation). A swept sine signal from a dynamic signal analyzer (35670A, Agilent Technologies) was fed into the power supply. The frequency responses of the output current of the power supply and the signal of the Hall probe were measured by the signal analyzer.

Figure 8 shows the Bode diagrams of the horizontal and vertical steering fields, which were measured with the signal of the Hall probe put on the center of steering aperture. The excitation current was set to be 1 A peak-to peak, that is a maximum current for fast orbit feedback operation. As shown in this figure, the steering system has good frequency response. The field attenuation due to the effect of eddy current on the bellows duct is insignificant.

When both the horizontal and vertical streeings are switched on, interference between the two steerings may occur due to mutual inductance of the coils. Figure 9 shows the measured Bode diagrams of the power supply output current for vertical steering with the horizontal steering on and off. In this measurement, two AC power supplies driven by the identical signal from the signal analyzer were used. The output current of them was $1 \mathrm{~A}$ peak-to peak. The result shows there is no difference between the cases horizontal steering on and off. It implies that the interference due to mutual inductance is negligible and the steering can be operated independently in the horizontal and vertical directions. (a)

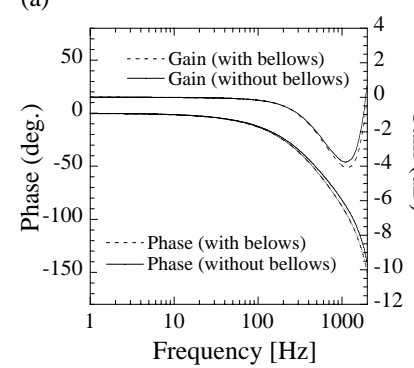

(b)

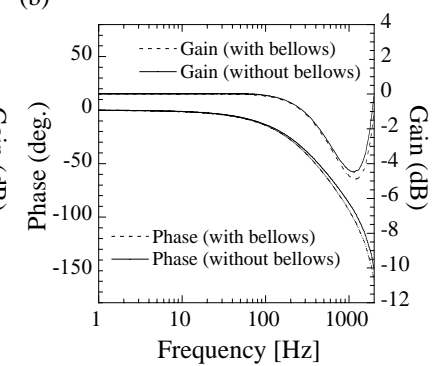

Figure 8: Frequency response of the (a) horizontal and (b) vertical fields.

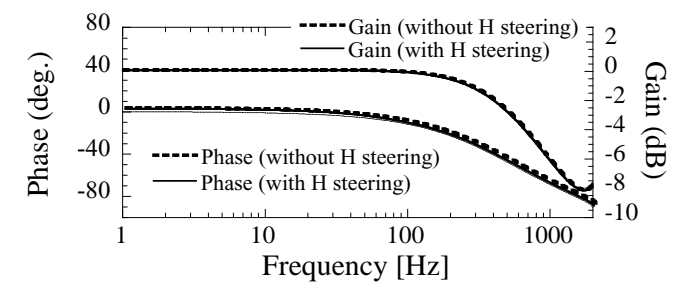

Figure 9: Frequency response of the output current of the vertical steering power supply.

\section{REFERENCES}

[1] Recently, the nickname of the project was changed from "VSX project" to "Super SOR project". The new name is associated with the Japanese first storage ring, SOR-RING, which was constructed in '70s and operated over 20 years as a frontier of the synchrotron radiation science in Japan.

[2] H. Takaki et al., "The Lattice of $1.0 \mathrm{GeV}-1.6 \mathrm{GeV}$ VSX Storage Ring", accepted for publication in Nucl. Instrum. Methods A.

[3] K. Harada et al., "The Effect of the Insertion Devices at the Super SOR Light Source," in these proceedings.

[4] N. Nakamura et al., Proc. EPAC 2000, Vienna, p. 669.

[5] Y. Hori et al., "Vacuum System Design and Research for the Super SOR", in these proceedings.

[6] M. Satoh et al., "Development of the Orbit Feedback System for the VSX Ring", accepted for publication in Nucl. Instrum. Methods A.

[7] T. Koseki et al., Proc. 1999 PAC, New York, p. 3363. 